- Case Report

\title{
A Case Report of Failure to Thrive Caused by Parental Intellectual Disability: The Invisible Population
}

\author{
Atirah Az-Zahrah Abu Bakar, Azidah Abdul Kadir*, Nursuhaila Idris \\ Department of Family Medicine, School of Medical Sciences, Health Campus, Universiti Sains Malaysia, Kubang Kerian, Malaysia
}

This case illustrates the medical complication of a child that arose as a consequence of parents with intellectual disabilities in a primary care setting. A 9-month-old girl presented with multiple admissions because of recurrent pneumonia and was later diagnosed with failure to thrive based on child neglect resulting from the parents' disabilities. This exemplifies a failure to thrive case with a complex and undiagnosed psychosocial background that required interdepartmental involvement to mitigate the health problem.

Keywords: Failure to Thrive; Parental Intellectual Disability; Child Abuse; Child Custody; Case Report

Received: April 1, 2020, Revised: April 7, 2020, Accepted: April 8, 2020

*Corresponding Author: Azidah Abdul Kadir https://orcid.org/0000-0003-1039-6304

Tel: +60-97676604/6608, Fax: +60-97676611,E-mail: azidahkb@usm.my 


\section{INTRODUCTION}

Parental intellectual disability has been discussed for many years, with affected parents subject to stigmatization and discrimination based on their inability to live normal lives. ${ }^{1)}$ They are considered less capable of practicing effective parenting skills, and this in turn has led to crucial issues related to their children's wellbeing. However, the future impact on children whose parents are intellectually challenged is still up for debate. $^{2)}$ These children are believed to be more vulnerable to abuse, neglect, mental health problems, disabilities, and poverty. As observed by primary care providers, failure to thrive is a consequence of one or more of the aforementioned factors. The presumption of inadequate parental competencies due to cognitive limitations-as evidenced by their low intelligence quotients-raises the issue of whether parents with intellectual disabilities should retain normal inalienable rights to parent or whether those rights should be revoked, especially in the event of maltreatment.

\section{CASE REPORT}

A 9-month-old girl presented with prolonged productive cough for 1 month and experienced frequent vomiting. She had a history of recurrent pneumonia, failure to thrive, and global developmental delay from the age of 2 months. However, her parents had failed to comply with follow-up sessions and treatment. Anthropometric measurements noted that the young child weighed $4.6 \mathrm{~kg}$ (below $3 \mathrm{rd}$ centile), her height was $60 \mathrm{~cm}$ (below 3rd centile) and her head circumference was $39 \mathrm{~cm}$ (10th centile). A physical assessment revealed minimal subcutaneous fat, reduced muscle mass, thin extremities, soft syndromic features with partial ptosis of the left eye, small chin, tonguetie, low hair amount, and dry skin. Examination of her respiratory system revealed reduced air entry on the left side. The child's bilateral upper and lower limbs showed hypertonia and hyperreflexia, and her developmental assessment showed slight delays in gross and fine motor components albeit with normal development of speech and social behavior. Blood investigations, urinalysis, and a chest X-ray were all unremarkable.

Upon further review of her social history, the patient was the second child of parents with intellectual disability. The father was illiterate with learning disabilities. The mother had underlying intellectual disability with a mental age of between 7-9 years, and she was currently under psychiatric follow-up care. The mother was known to have exhibited abnormal behavior a few hours after giving birth to her daughter. She was assessed by a psychiatric team and was appropriately diagnosed with adjustment disorder. A memorandum regarding her condition was submitted by the tertiary center to the mother's primary physician in order to alert the physician of their intensive observation of both the mother and the patient after they were discharged from the postnatal ward. Subsequently, the mother was frequently observed inflicting harm on her child.

The couple's first child was a 3-year-old boy. He had been in the custody of his paternal uncle from the age of 2 to 6 months after the father's family decided to separate the first child from his parents for a short period because of the harm inflicted by the mother. They did not report the event to the welfare department nor the child protection services. However, from the age of 6 months, the first child received care from his parents with additional supervision from their extended families. The boy appeared to be thriving with no medical or developmental concerns.

The girl in this case was admitted for pneumonia and discharged to return home after her condition improved. During a clinical follow-up, it was noted that she had been taken from her mother by the child protection agency after they received a report that the mother was attempting to harm the child. At that point, the child was placed under the custody of her father with additional supervision provided by her paternal uncle and family. Meanwhile, the mother was placed under the care of her biological parents.

After this change in living arrangements, the girl showed significant improvement. Her growth chart indicated a weight gain from minus 3 standard deviation (SD) to within the normal range (0 to minus 2SD). At 15 months of age, she was returned to the care of both of her parents with additional collaborative supervision from the paternal uncle. Financially, both parents were receiving monthly allowances from Welfare Department.

Written informed consent regarding the publication of clinical details related to this case report was obtained from the parents of the patient.

\section{DISCUSSION}

Intellectual disability is a disorder characterized by deficits in intellectual and adaptive functioning that begins during the developmental period. Those diagnosed with this disorder can be categorized into four degrees of severity: mild, moderate, severe, or profound based on their performance in the conceptual, social, and practical domains. ${ }^{3)}$ The actual prevalence of parents with intellectual disability remains undetermined. ${ }^{4)}$ They are reported to be adversely affected by numerous disadvantages related to health issues, socioeconomic factors, and restricted social networks. As with the normal population, many individuals with intellectual disabilities still choose to be parents despite their circumstances and limitations.

However, many intellectually challenged parents are either barely or totally unaware that their children will face higher risks of experiencing developmental delays and behavioral issues along with speech and language difficulties. Their children are also reported to have a much higher risk of accidents and injuries.5 ${ }^{5}$ An acute lack of stimulation and insufficient social support from intellectually disabled parents will significantly influence the children's developmental outcomes. Moreover, children of intellectually impaired mothers are at greater risk of poor social-emotional wellbeing. ${ }^{6}$ )

Exploring the essential needs and experiences of intellectually disabled parents will help primary care providers assist them in caring for 
their children. ${ }^{7)}$ The first stage is to identify the parental communication, social skills, and existing networks to begin conditioning them to accept suggestions and improve their social support. ${ }^{8)}$ However, based on their limited intelligence and the difficulties they face when trying to interpret instructions and suggestions, they require substantial formal and informal support. Their children should be provided with diverse types of informal support, especially from other family members and relatives, while formal support can be augmented through the guidance and supervision offered by social workers and welfare staff.9) Additionally, medical advice should be available to family members to ensure the provision of information and related interventions. Other options to guarantee that their children receive adequate nutrition, stimulation, affectionate care, proper medical attention, and protection against unintentional injury or accidents may be facilitated by their willingness to entrust eligible family members with the temporary custody of their children.

As revealed in this case report, the patient failed to thrive because of unintentional neglect by her intellectually disabled parents. Child neglect is one of the leading causes of nonorganic failure to thrive. It requires integrated collaborative management involving professionals from several health disciplines, including pediatricians, psychiatrists, family medicine practitioners, dieticians, and physiotherapists. Holistic management should involve the patient, parents or guardians with their extended families, the primary health care provider, and additional health care professionals. The principle goal of treatment is to establish an optimal growth rate by supporting the family in their efforts to develop and adhere to an appropriate care plan. Regarding the specific role of family physicians, albeit secondary to child neglect, cases of failure to thrive also require thorough evaluations of each risk factor pertaining to specific familial situations. Home visits can be conducted to complete or validate clinical evaluations and assess the social aspects and living environment of the patient.

When child neglect is suspected as a cause of any medical issue, the treating doctor is obligated to submit a formal report to the welfare department whose involvement is fundamental for establishing the family's social status and revealing underlying issues that may have led to the neglect. Child protection officers will normally proceed with a meticulous investigation to decide whether qualified personnel should be appointed as the child's legal guardian if the parents or current guardian are unable to provide adequate safety and care. Medical doctors are called upon to assess the mental state, health awareness, and the capacity of the guardian to follow appropriate medical advice. In circumstances where the nearest family members are unable to help care for the neglected child, the welfare department is obligated to provide a suitable nursing home. In this case report, the patient clearly exhibited significant improvement in nutritional status as well as her behavioral and emotional wellbeing after being placed in her father's custody that included comprehensive supervision by her paternal uncle and aunt. In sum, this case verified that the mother was too incompetent to raise the child.

All pregnancies among mothers with intellectual impairment should be planned and intentional. Any possibly related complications during the pregnancy, intrapartum, and postpartum periods must be fully explained. Other predicaments associated with the medical and psychosocial conditions of children burdened with parental intellectual disability should also be highlighted, and these discussions should involve the parents' family members. Prior to this, holistic assessments regarding the competence of mothers to raise their babies can be conducted, and the severity level of intellectual disability generally determines an individual prognosis for each child.

After the child in this case was delivered, the tertiary center promptly informed the primary care physician regarding the possibility of neglect based on parental intellectual disability. However, further followup sessions were not properly conducted. Although the child showed poor weight gain during her first 6 months, no intensive intervention was scheduled. Feeding issues and the inability of the parents to acknowledge basic feeding cues should be further evaluated. As the mother exclusively breastfed her baby, a comprehensive assessment of her breastfeeding technique, feeding position, and the presence of breast or nipple complications remains a matter of contention. Primary care providers are the health system's front line; therefore, the training and preparation for their roles must be enhanced by providing the knowledge necessary to adequately manage medical consequences related to parental intellectual disability.

Early comprehensive evaluations regarding the home atmosphere, communication, and interaction between the patient and the familyas well as early detection of the parents' inability to establish a proper feeding plan-will lead to corrective measures that can prevent the occurrence of failure to thrive and mitigate subsequent health problems. It is also important to encourage extended family members to monitor individuals with intellectual disability while encouraging them to seek help for their social, emotional, financial, and interpersonal problems, the mitigation of which could reduce the incidence of psychosocial failure to thrive. ${ }^{10)}$

The numerous challenges encountered by parents with intellectual disability raise the question of whether the parents in this specific case report were qualified to retain their parental rights. Despite specific education and supervision, the parents' ability to provide adequate care, emotional support, and sufficient stimulation as required by the child remains controversial and undetermined. To answer this question, a joint effort between healthcare providers, the welfare department, and child protection services should be undertaken to assess the parents and family. This type of profound evaluation must be performed continuously. Despite intensive and holistic approaches from the aforementioned agencies, parents with intellectual disabilities still face hardships when trying to meet the needs of their children. Future consequences suffered by these children include increased susceptibility to separation from their parents and either short-term or permanent placement in nursing or foster homes. ${ }^{\text {g) }}$ 


\section{CONFLICT OF INTEREST}

No potential conflict of interest relevant to this article was reported.

\section{ORCID}

Atirah Az-Zahrah Abu Bakar: https://orcid.org/0000-0003-4402-2706

Azidah Abdul Kadir: https://orcid.org/0000-0003-1039-6304

Nursuhaila Idris: https://orcid.org/0000-0002-5877-061X

\section{REFERENCES}

1. LaLiberte T, Piescher K, Mickelson N, Lee MH. Child protection services and parents with intellectual and developmental disabilities. J Appl Res Intellect Disabil 2017;30:521-32.

2. Collings S, Llewellyn G. Children of parents with intellectual disability: facing poor outcomes or faring okay? J Intellect Dev Disabil 2012;37: 65-82.

3. American Psychiatric Association. Desk reference to the diagnostic criteria from DSM-5. Washington (DC): American Psychiatric Associa- tion; 2013.

4. Powell RM, Parish SL. Behavioural and cognitive outcomes in young children of mothers with intellectual impairments. J Intellect Disabil Res 2017;61:50-61.

5. Emerson E, Brigham P. The developmental health of children of parents with intellectual disabilities: cross sectional study. Res Dev Disabil 2014;35:917-21.

6. Hindmarsh G, Llewellyn G, Emerson E. The social-emotional well-being of children of mothers with intellectual impairment: a populationbased analysis. J Appl Res Intellect Disabil 2017;30:469-81.

7. Powell RM, Parish SL, Akobirshoev I. Health of young children whose mothers have intellectual disability. Am J Intellect Dev Disabil 2016; 121:281-94.

8. Llewellyn G, Hindmarsh G. Parents with intellectual disability in a population context. Curr Dev Disord Rep 2015;2:119-26.

9. Gudkova T, Hedlund M, Midjo T. Supporting children of parents with intellectual disability: a scoping review. J Appl Res Intellect Disabil 2019;32:737-49.

10. Onyiriuka AN. Evaluation and management of the child with failure to thrive. Hosp Chron 2011;6:9-23. 